\title{
Physikalische Therapie: Therapiemittel Licht (1)
}

\author{
Jean-Michel Jeannin
}

Basel, Schweiz

\section{Einleitung}

Natürliches und künstliches Licht wird zur Behandlung von Depressionen, saisonalen affektiven Störungen und Schlafstörungen eingesetzt. Die Phototherapie von Dermatosen wird in einer weiteren Folge dieser Rubrik behandelt.

\section{Biologischer Tagesrhythmus}

Der biologische Tagesrhythmus wird massgeblich vom Licht bestimmt. Die Netzhaut des Auges enthält lichtempfindliche (photosensible), nicht bildgebende Ganglienzellen [1]. Sie leiten den Reiz direkt an Taktgeber des circadianen Rhythmus im Hypothalamus, einer Struktur des Gehirns, die das vegetative Nervensystem steuert. Das von den photosensiblen Ganglienzellen produzierte Melanopsin bewirkt eine lichtabhängige Unterdrückung der Ausschüttung von Melatonin (Abb. 1). Melatonin seinerseits wirkt schlafanstossend. Der biologische Tagesrhythmus kann mittels Lichtexposition beeinflusst werden. Am meisten wirksam scheint Licht mit einer Temperatur von $6500 \mathrm{~K}$ zu sein (siehe Kasten): Eine Lichtmenge von $40 \mathrm{~lx}$ ist dazu ausreichend. Eine solche Exposition lässt sich mit handelsüblichen Neonröhren erzielen [2].

\section{Saisonale affektive Störung («Winterdepression»)}

Als Ursache wird eine Störung des biologischen Tagesrhythmus im Sinne einer Verspätung oder Vorverlegung einer Phase angenommen, die ihrerseits auf einem Lichtmangel beruht [3]. Lichtmangel ist möglicherweise mit einem Serotoninmangel vergesellschaftet [3-5]. Der Melatonin-Spiegel war von einer Lichtexposition weder bei Patienten noch bei Versuchspersonen verändert, im Un-
Kasten. Was man vom Licht wissen muss.

- Wellenlänge $\lambda(\mathrm{nm})$ : Das Spektrum des für den Menschen sichtbaren Lichts reicht von etwa $380 \mathrm{~nm}$ (Violett) bis circa $720 \mathrm{~nm}$ (Rot). Von der Wellenlänge hängt $\mathrm{ab}$, welche Farbe wir wahrnehmen.

- Kelvin (K): Farbtemperatur einer Lichtquelle. Stellt ein Mass für den Farbeindruck dar. Weisses Licht hat die Temperatur von circa $5500 \mathrm{~K}$. Die Bestimmung der Farbtemperatur dient dem technischen Vergleich verschiedener Lichtquellen.

- Candela (cd): Einheit für die Lichtstärke.

- Lumen ( $\mathrm{lm}$ ): Einheit für den Lichtfluss (die von einer Lichtquelle ausgestrahlte Lichtmenge pro Zeit).

- Lichtstärke Lux (lx): Mass für die einfallende Lichtmenge pro Fläche.

Abb. 1. Melatonin.<smiles>COc1ccc2[nH]cc(CCNC(C)=O)c2c1</smiles>

terschied zum Serotonin-Spiegel, der nach der Exposition mit hellem und mit gedämpftem Licht erhöht war (Abb. 2, 3) [4]. Von einer Winterdepression sind in unseren Breitengraden etwa $4-10 \%$ der Bevölkerung betroffen, in nördlicheren Gegenden mehr, in südlicheren weniger [3, 5]. Betroffene leiden während rund $40 \%$ der Zeit an den Symptomen. Eine saisonale affektive Störung tritt typischerweise nach der Herbst-Tagundnachtgleiche auf und bildet sich nach der Frühjahrs-Tagundnachtgleiche spontan zurück. Neben den typischen Symptomen einer Depression (Traurigkeit, Ängstlichkeit, Energieverlust) werden auch eine Zunahme des Appetits (vornehmlich auf Kohlenhydrate) sowie des Gewichts beobachtet (als Ausdruck eines Serotoninmangels), ferner Mühe bei der Arbeit und im zwischenmenschlichen Umgang [3, 6]. Eine Phototherapie ist eine kausale Therapie der Winterde-

\section{KARGER \\ Fax +497614520714} Information@Karger.com www.karger.com 


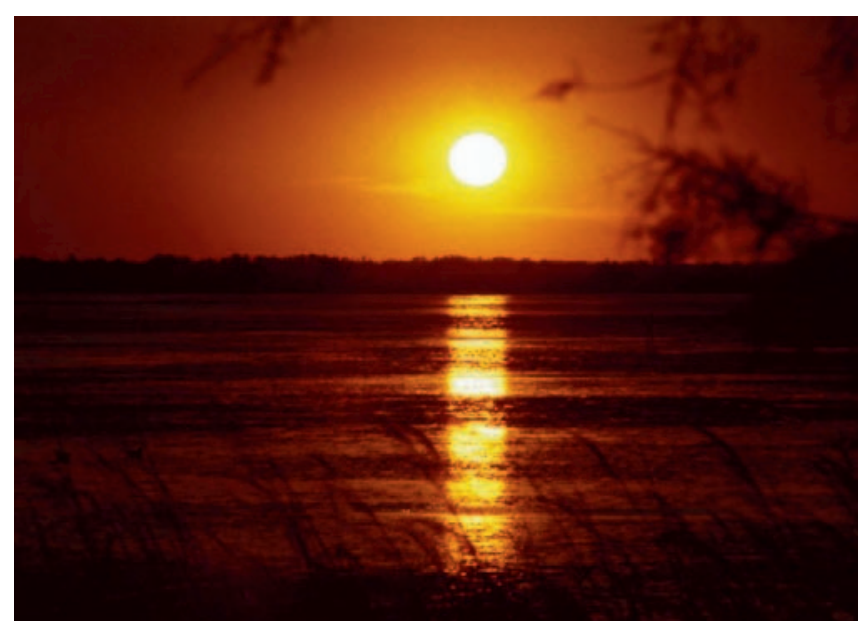

Abb. 2. Die beste Lichtquelle: Sonne.

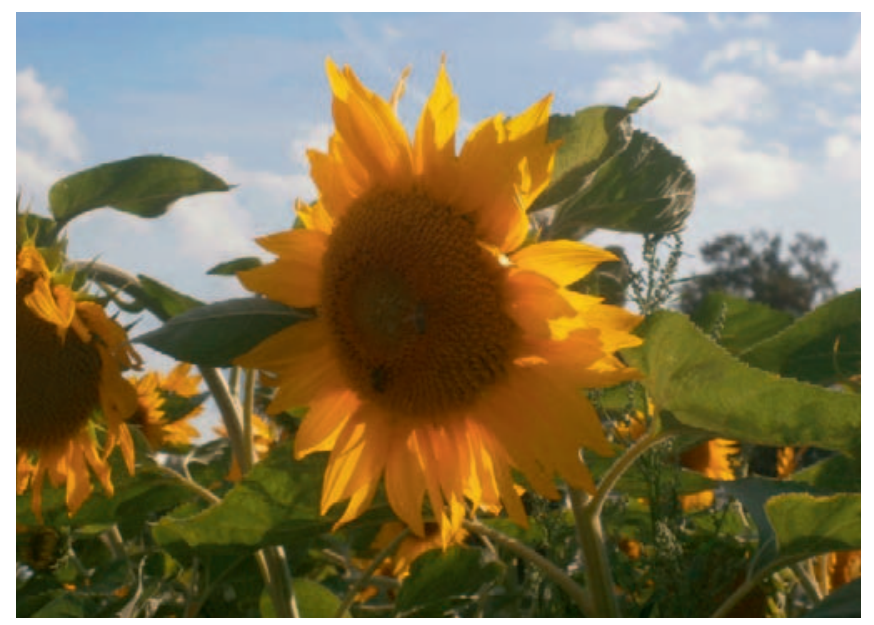

Abb. 3. Sonnenblume (Helianthus annuus).

pression. Anwendungen mit hellem Licht (3300 lx), mittelhellem Licht und gedämpftem Licht zeigten einen guten Behandlungserfolg [3]. Gemäss Kurlansik und Ibay [5] ist die Exposition am frühen Morgen mit einem Abstand von $30-45 \mathrm{~cm}$ zu einer weissen Lichtquelle ohne UV-Anteil mit einer Stärke von $10000 \mathrm{~lx}$ während $30 \mathrm{~min}$ täglich am besten wirksam. Zu einem vergleichbaren Ergebnis kommen Terman et al. [6]: Die beste Lichtstärke war 2500 lx; die beste Wirkung zeigte die Behandlung am Morgen (53\% Reduktion auf der Hamilton-Skala), gefolgt von der Behandlung am Abend (38\% Reduktion) und der Behandlung am Mittag (32\% Reduktion). Es bleibt zu berücksichtigen, dass eine Minderheit der Patienten auf eine abendliche Behandlung besser anspricht als auf eine morgendliche. Der Behandlungserfolg war ferner bei leichten Fällen am besten. Lichttherapie gilt als eine sichere Behandlung. Mögliche Nebenwirkungen sind eine Hypomanie, Reizbarkeit, Kopfschmerzen und Übelkeit. Die Kopfschmerzen gehen nach einer Dosisreduktion zurück [7]. Der beste Tageszeitpunkt für den Erfolg einer Photothe-

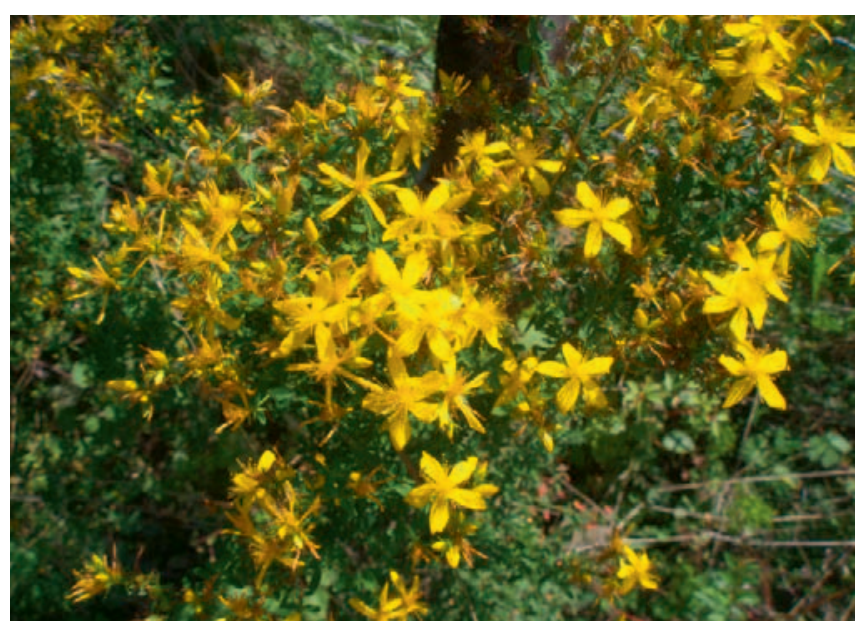

Abb. 4. Hilft wie das Licht bei Depression: Johanniskraut.

rapie hängt wesentlich vom Chronotypen des Patienten ab: Frühaufsteher werden um 7.30 Uhr, Spätaufsteher um 8.30 Uhr am effizientesten behandelt. Der Chronotyp kann mittels Fragebogen ermittelt werden [8]. Da keine Placebo-Behandlung möglich ist, ist eine genaue und differenzierte Beurteilung der Lichttherapie bei saisonaler affektiver Störung schwierig [6].

\section{Nichtsaisonale Depression}

Der Zusammenhang zwischen einem Licht- und einem Serotoninmangel bildet die Grundlage für die Phototherapie der nichtsaisonalen Depression (Abb. 4). Die Wirkung des Antidepressivums Citalopram bei der Behandlung von an einer Major Depression oder an einer bipolaren Depression Erkrankten wurde durch die Kombination mit einer morgendlichen Phototherapie verbessert [9]. Wirz-Justice und Staedt empfehlen die Lichttherapie zur Behandlung der Major Depression sowie der Depression nach Schlaganfall [8]. Eine spezielle Form der Lichttherapie ist der Schlafentzug. Ein Schlafentzug ist bei einem breiten Spektrum von depressiven Störungen wirksam, allerdings in unterschiedlichem Mass: Patienten mit einer endogenen Depression sprechen häufiger an $(75 \%$; $68 \%)$ als jene mit einer neurotischen Depression (48\%; 33\%). Ebenso profitieren Patienten mit einer bipolaren Störung mehr von einer Therapie als solche mit einer unipolaren Depression. Ein Schlafentzug wirkt auf Frauen und Männer gleich gut. Die Wirkung eines Schlafentzugs ist am darauffolgenden Tag am grössten. Das Ausbleiben einer Wirkung bedeutet nicht, dass der betreffende Patient auch auf eine weitere Therapie nicht anspricht. Eine Schlafentzugstherapie wird durch eine begleitende Pharmakotherapie nicht verbessert. Umgekehrt verbessert eine Schlafentzugstherapie die Wirksamkeit einer Pharmakotherapie [10]. 


\section{Schlafstörungen und weitere Anwendungsmöglichkeiten der Lichttherapie}

Schlafstörungen, die sich für eine Lichttherapie eignen, sind solche, die auf einer Verschiebung der Einschlafzeit beruhen, d.h. vorverlagertes Schlafphasensyndrom oder verzögertes Schlafphasensyndrom. Mit einer Lichttherapie wird die verlagerte Schlafphase rück- oder vorverlagert. Mittels einer Lichttherapie nach dem Aufstehen, die schrittweise vorgelegt wird, wird eine verzögerte Schlafphase vorverlegt. Umgekehrt wird mit einer Therapie am frühen Abend, die schrittweise nach später verschoben wird, eine vorverlagerte Schlafphase wieder rückverlagert, d.h. auf später verschoben [11]. Eine Lichttherapie eignet sich ferner zur Behandlung des Jetlags und von Schlafstörungen infolge von Schichtarbeit [12]. Eine Lichttherapie kann ferner zur Synchronisation eines freilaufenden
Schlaf-Wach-Rhythmus bei Sehbehinderten verabreicht werden, ebenso bei Bulimie oder einer Aufmerksamkeitsdefizit-/Hyperaktivitätsstörung [11].

\section{Schlussbemerkungen}

Das Therapiemittel Licht kann vielfältig eingesetzt werden. Viele damit behandelten Störungen und Krankheiten stehen in einem gewissen Zusammenhang mit der modernen Lebensweise, die auf natürliche Rhythmen nur wenig Rücksicht nimmt. Licht wird oft als natürliches Heilmittel bezeichnet und bisweilen mit Phytotherapie in einem genannt. Eine bessere Beachtung der natürlichen Rhythmen verhütet manche Störung und ist garantiert frei von unerwünschten Wirkungen.

\section{Literatur}

1 Fotosensitive Ganglienzelle. Wikipedia. https://de.wikipedia.org/wiki/Fotosensitive Ganglienzelle (aufgerufen am 24.08.2015).

2 Chellappa SL, et al: Non-visual effects of light on melatonin, alertness and cognitive performance: can blue-enriched light keep us alert? PLoS One 2011;6:e16429.

3 Miller AL: Epidemiology, etiology, and natural treatment of seasonal affective disorder. Altern Med Rev 2005;10:5-13.

4 Rao ML, et al: Blood serotonin, serum melatonin and light therapy in healthy subjects and in patients with nonseasonal depression. Acta Psychiatr Scand 1992;86:127-132.
5 Kurlansik SL, Ibay AD: Seasonal affective disorder. Am Fam Physician 2012;11:1037-1041

6 Terman M, et al: Light therapy for seasonal affective disorder. A review of efficacy. Neuropsychopharmacology 1989;2:1-22.

7 Terman M, Terman JS: Light therapy for seasonal and nonseasonal depression: efficacy, protocol, safety, and side effects. CNS Spectr 2005; 10:647-663.

8 Wirz-Justice A, Staedt J: Lichttherapie - nich nur bei Winterdepression. Psychiatrie 2008; 25-31.
9 Benedetti F, et al: Morning light treatment hastens the antidepressant effect of citalopram. J Clin Psychiatry 2003;64:648-653.

10 Wirz-Justice A, Van den Hoofdacker RH: Sleep deprivation in depression: what do we know, where do we go? Biol Psychiatry 1999; 46:445-453.

11 Wirz-Justice A, Bromundt V: Lichttherapie. Schlaf 2003;1:20-29.

12 Gooley JJ: Treatment of circadian rhythm sleep disorders with light. Ann Acad Med Singapore 2008;37:669-676. 